\title{
Prospective, Double-Blind, Randomized, Placebo-Controlled Comparison of Acetazolamide Versus Ibuprofen for Prophylaxis Against High Altitude Headache: The Headache Evaluation at Altitude Trial (HEAT)
}

Jeffrey H. Gertsch, MD; Grant S. Lipman, MD; Peter S. Holck, PhD; Andrew Merritt, MD; Allison Mulcahy, MD; Robert S. Fisher, MD, PhD; Buddha Basnyat, MD; Eric Allison, DO; Keeli Hanzelka, MD; Alberto Hazan, MD; Zachary Meyers, MD; Justin Odegaard, MD, PhD; Benjamin Pook, MBChB; Mark Thompson, MD; Brant Slomovic, MD; Henrik Wahlberg, MBChB; Vanessa Wilshaw, MBChB; Eric A. Weiss, MD; Ken Zafren, MD

From the Department of Neurosciences, University of California-San Diego School of Medicine, San Diego, CA (Dr Gertsch); Department of Surgery, Stanford University School of Medicine, Division of Emergency Medicine, Palo Alto, CA (Drs Lipman, Weiss, and Zafren); Department of Public Health Sciences and Epidemiology, John A. Burns School of Medicine, University of Hawaii, Honolulu, HI (Dr. Holck); Keck School of Medicine, University of Southern California, Los Angeles, CA (Dr. Merritt); Department of Emergency Medicine, Highland General Hospital, Oakland, CA (Dr Mulcahy); Department of Neurology, Stanford University School of Medicine, Palo Alto, CA (Dr Fisher); Nepal International Clinic, Kathmandu, Nepal (Dr Basnyat); Arizona College of Osteopathic Medicine, Phoenix, AZ (Dr Allison); Emergency Service Partners, La Costa Centre, Austin, TX (Dr Hanzelka); Department of Emergency Medicine, St. Luke's Hospital, New York, NY (Dr Hazan); Montana Family Medicine Residency, Billings, MT (Dr Meyers); Stanford University School of Medicine, Multidisciplinary Program in Immunology, Stanford, CA (Dr Odegaard); Royal College of Surgeons Ireland, Dublin, Ireland (Drs Pook and Wahlberg); Department of Emergency Medicine, Advocate Christ Medical Center, Oak Lawn, IL (Dr Thompson); Department of Emergency Medicine, University Health Network, Toronto, Ontario, Canada (Dr Slomovic); Leicester Medical School, University of Leicester, Leicester, England (Dr Wilshaw).

Objective.-High altitude headache (HAH) is the most common neurological complaint at altitude and the defining component of acute mountain sickness (AMS). However, there is a paucity of literature concerning its prevention. Toward this end, we initiated a prospective, double-blind, randomized, placebo-controlled trial in the Nepal Himalaya designed to compare the effectiveness of ibuprofen and acetazolamide for the prevention of HAH.

Methods.-Three hundred forty-three healthy western trekkers were recruited at altitudes of $4280 \mathrm{~m}$ and $4358 \mathrm{~m}$ and assigned to receive ibuprofen $600 \mathrm{mg}$, acetazolamide $85 \mathrm{mg}$, or placebo 3 times daily before continued ascent to $4928 \mathrm{~m}$. Outcome measures included headache incidence and severity, AMS incidence and severity on the Lake Louise AMS Questionnaire (LLQ), and visual analog scale (VAS).

Results.-Two hundred sixty-five of 343 subjects completed the trial. HAH incidence was similar when treated with acetazolamide $(27.1 \%)$ or ibuprofen $(27.5 \% ; P=.95)$, and both agents were significantly more effective than placebo $(45.3 \% ; P=.01)$. AMS incidence was similar when treated with acetazolamide $(18.8 \%)$ or ibuprofen $(13.7 \% ; P=.34)$, and both agents were significantly more effective than placebo $(28.6 \% ; P=.03)$. In fully compliant participants, moderate or severe headache incidence was similar when treated with acetazolamide $(3.8 \%)$ or ibuprofen $(4.7 \% ; P=.79)$, and both agents were significantly more effective than placebo $(13.5 \% ; P=.03)$.

Conclusions.-Ibuprofen and acetazolamide were similarly effective in preventing HAH. Ibuprofen was similar to acetazolamide in preventing symptoms of AMS, an interesting finding that implies a potentially new approach to prevention of cerebral forms of acute altitude illness.

Key words: altitude, headache, ibuprofen, acetazolamide, acute mountain sickness, high altitude headache

Corresponding author: Jeffrey H. Gertsch, MD, Assistant Clinical Professor, Director, Interventional Neurophysiology, Department of Neurosciences, University of California-San Diego School of Medicine, 200 West Arbor Dr, Mail Code \#8465, San Diego, CA 921038465 (e-mail: jgertsch@ucsd.edu).

\section{Introduction}

Headache ranks among the most common complaints at altitude, with an incidence of $25 \%$ at $1920-2956 \mathrm{~m}$ to between $47 \%$ and $62 \%$ at $4928 \mathrm{~m}^{1-4}$ Diagnostic criteria have been proposed for altitude-associated headaches 
(termed high altitude headache or HAH) by researchers and expert panels; however, HAH is sufficiently variable in presentation that altitude researchers mainly define HAH by the setting under which it occurs..$^{5-7}$

$\mathrm{HAH}$ frequently occurs as a benign isolated ailment but is also considered a sentinel symptom in the development of acute mountain sickness (AMS). HAH is the requisite cornerstone symptom of AMS, defined at altitudes above $2500 \mathrm{~m}$ by the presence of headache and at least 1 other symptom of anorexia, fatigue, insomnia, or dizziness. If unrecognized or left untreated, advanced AMS may progress to its end-stage expression as high altitude cerebral edema. The diagnosis of high altitude cerebral edema comes with significant mortality, highlighting the practical importance of preventing HAH and AMS at an early stage. ${ }^{8-10}$

Ibuprofen is a widely available over-the-counter, nonsteroidal anti-inflammatory drug (NSAID) commonly used for treatment of HAH. Ibuprofen via cyclo-oxygenase inhibition prevents the production of prostaglandins and the inflammatory cascade, chemical irritants known to sensitize meningovascular receptors that mediate nociception. ${ }^{10-14}$ Multiple studies show the NSAID aspirin to be an effective prophylactic agent against HAH as well. ${ }^{15-17}$ Several studies put forth conflicting data with respect to NSAIDs' ability to prevent AMS. Two small studies have suggested that the related NSAIDs naproxen and calcium carbasalate are ineffective for prevention of AMS, whereas a preliminary observational report suggests a protective effect. ${ }^{18-20}$

Acetazolamide is a diuretic and carbonic anhydrase inhibitor used as the standard prophylactic agent against AMS, and has been documented to prevent headache at altitude as well..$^{2-4,21}$ Acetazolamide's multifactorial mechanism of prevention for altitude sickness includes renally induced metabolic acidosis resulting in diuresis and enhanced ventilation, improvements in sleep quality from modulation of carotid body activity, and inhibition of cerebrospinal fluid production. ${ }^{22,23}$ While intuitive that acetazolamide would prevent headaches at altitude, it has seen limited study for HAH prevention specifically. ${ }^{16}$

This study was designed to compare the effect of the NSAID ibuprofen with acetazolamide for prevention of HAH and AMS, as well as to better establish clinical standards for HAH prevention.

\section{Methods}

This study was designed as a prospective, double-blind, randomized, placebo-controlled trial. Enrollment took place between October and November 2005 along the approach trail to Mount Everest in the Nepali Himalaya. The study was conducted in accordance with the Decla- ration of Helsinki, ${ }^{24}$ and under the auspices of the Himalayan Rescue Association. Ethical approval was provided by the Stanford University School of Medicine Institutional Review Board and the Nepal Health Research Council.

\section{Participants and Outcome Measures}

Trekkers completed questionnaires after giving signed informed consent. Inclusion criteria specified healthy non-Nepali males and females 18 to 65 years of age traveling directly between the baseline villages of Pheriche or Dingboche $(4280 \mathrm{~m}$ and $4358 \mathrm{~m}$, respectively) and the endpoint in Lobuje (4928 m). Potential participants were excluded if they had any headache, diagnosis of AMS, signs or symptoms of a substantial acute infection, had slept above $4500 \mathrm{~m}$, or had taken any NSAIDs or acetazolamide within 1 day or 3 days prior to enrollment, respectively.

The predetermined primary outcome measure was presence of headache incidence at the study endpoint as calculated on the Lake Louise AMS Questionnaire (LLQ), a validated field standard for diagnosis of AMS, which includes a question on headache presence and severity. ${ }^{25,26}$

A predetermined secondary endpoint included evaluation of headache severity by Visual Analog Scale (VAS). ${ }^{27,28}$ Other secondary measures included pulse oximetry (Nonin Medical Products, Minneapolis, MN), as well as AMS incidence and severity as measured by the LLQ. Demographics, ascent profile, compliance, and side effects data were collected to adjust for potential confounders.

\section{Study Design}

Commercial pharmaceutical grade acetazolamide and ibuprofen were packed in visually identical capsules by Deurali-Janta Pharmaceuticals (Kathmandu, Nepal). Study medications were randomized via computergenerated code. Participants were sought out on a daily basis in all baseline village hotels and sequentially enrolled in order to minimize selection bias.

All trekkers newly arrived at the baseline altitude were screened daily. Interviewers gathered demographics, ascent profile data, LLQ, VAS, and pulse oximetry. All trekkers were given information on methods for reducing the risk of AMS, thereby meeting the minimum standard of care. They were then randomized in a double-blind fashion to receive 3 times daily dosing of placebo, ibuprofen $600 \mathrm{mg}$, acetazolamide $85 \mathrm{mg}$ (total daily dose of $255 \mathrm{mg}$ to approximate a cumulative $250 \mathrm{mg}$ daily dose given for AMS prophylaxis). Participants took a mini- 
mum of 3 doses at the baseline altitude before proceeding on their trek.

On their ascent from baseline, a minority of participants stopped overnight at a lodge at $4595 \mathrm{~m}$, but all were expected to arrive at the endpoint altitude for data collection. VAS and LLQ scores were self-reported the night of arrival and the morning after arrival, at which point the study was complete. Endpoint data collection represents morning-after-arrival data (unless missing, then replaced with night-before data) in order to emphasize specificity in the diagnosis of HAH and AMS.

In order to minimize morbidity from high altitude illness, participants were discouraged, but not actively prevented, from using unblinded analgesics or acetazolamide. In the event of a severe illness that might be attributable to altitude or a reaction to medications, administrators were at the baseline and endpoint sites with appropriate steroid medication available.

\section{Statistical Analysis}

Power calculations suggested target sample sizes of 200 participants per treatment group and 100 in the placebo group. This was calculated to be sufficient to identify a statistically significant difference (at $80 \%$ power) between treatment and placebo, based on published HAH incidence of $50 \%$ at the study endpoint. ${ }^{2,4}$ Because the efficacy of acetazolamide relative to placebo has previously been established at identical altitudes and to permit improved power to detect differences between treatment groups, the relative number of participants allotted to the placebo group was reduced.

Intent-to-treat analyses, univariate comparisons (Pearson chi-square, Fisher exact tests, $t$ tests), and logistic regression were used to examine multivariate risk factors for binary outcomes. $P$ values less than .05 were considered significant. All analyses were conducted using $\mathrm{R}$ 2.6.0 software. $^{29}$

\section{Results}

A total of 343 healthy trekkers were enrolled and compared at baseline with 265 subjects presenting at the endpoint to provide data (78 subjects or $22.7 \%$ lost to follow-up, see Figure). An intent-to-treat analysis per protocol was used to evaluate the data, with 48 participants deviating from the proscribed protocol (see Table 1 and Figure).

While strict blinding and randomization methodology were incorporated, univariate comparisons of groups at baseline suggests that participants in the ibuprofen group were more likely to ascend slowly and sleep at the slightly higher baseline village of Dingboche (see Table

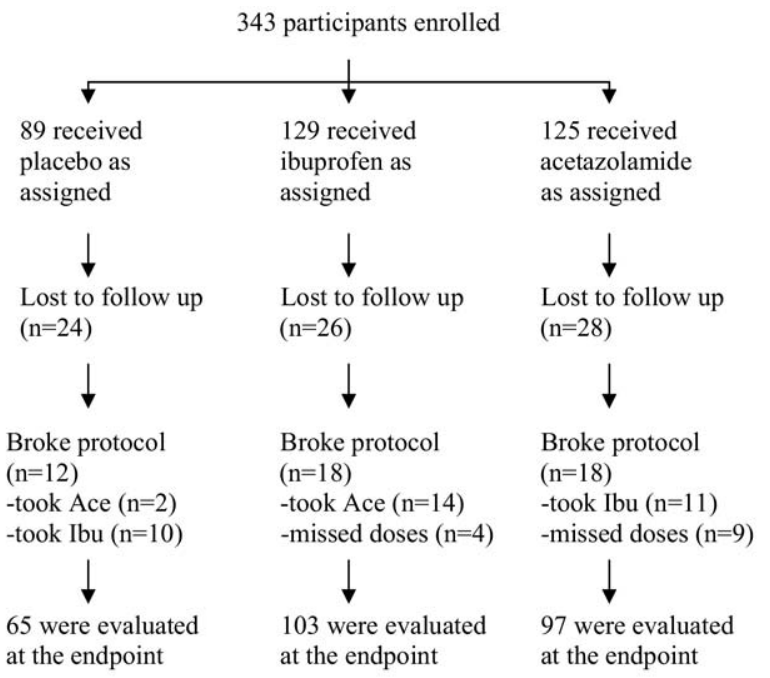

*Ace denotes acetazolamide, Ibu denotes ibuprofen.

Figure. HEAT study flow chart.

1), characteristics that could potentially provide a protective effect against altitude. As such, we included ascent rate and baseline village variables in our multivariate models to adjust for any potential effects. Neither variable approached significance in multivariate models (data not shown).

There were no major adverse events reported throughout the trial (ie, drug reactions, gastrointestinal bleeding, or life-threatening altitude sickness). Ibuprofen was not associated with a higher incidence of gastrointestinal upset or nausea after multiple statistical evaluations were performed.

\section{Intent-to-Treat Analysis}

Intent-to-treat data analysis revealed several key findings (see Table 2). For the primary endpoint, there was no significant difference between treatments in preventing HAH incidence (acetazolamide 27.1\%, ibuprofen 27.5\%; $P=.95)$. Combining treatment groups revealed a decrease in HAH incidence when compared to placebo at $45.3 \%(P=.01)$, resulting in a number needed to treat of 5.5. Headache severity was not significantly reduced either between treatments or compared to placebo by several measures (see below). The combined treatment groups were efficacious in prevention of AMS incidence (acetazolamide $18.8 \%$, ibuprofen $13.7 \%$, placebo $28.6 \%$, $P=.03)$, resulting in a number needed to treat of 8.1 . Participants taking placebo had a greater oxygen desaturation on ascent than those in the treatment arms $(P=.03)$.

\section{Secondary Analysis}

Analysis with fully compliant participants (defined as those who took all study medications and did not take off-study 
Table 1. Baseline characteristics of the completed study group

\begin{tabular}{|c|c|c|c|c|c|c|c|c|c|}
\hline \multirow[b]{2}{*}{ Cohort at endpoint (of 343$)^{a}$} & \multicolumn{2}{|c|}{$\begin{array}{c}\text { Study } \\
\text { participants }\end{array}$} & \multicolumn{2}{|c|}{ Placebo group } & \multicolumn{2}{|c|}{$\begin{array}{c}\text { Acetazolamide } \\
\text { group }\end{array}$} & \multicolumn{2}{|c|}{ Ibuprofen group } & \multirow[t]{2}{*}{$P$} \\
\hline & 265 & & 65 & $24.5 \%$ & 97 & $36.6 \%$ & 103 & $38.9 \%$ & \\
\hline Male gender & 187 & $71.1 \%$ & 47 & $72.3 \%$ & 65 & $67.7 \%$ & 75 & $73.5 \%$ & .64 \\
\hline Mean age & 38.3 & \pm 11.8 & 39.2 & \pm 12.1 & 39.1 & \pm 12.0 & 37 & \pm 11.4 & .36 \\
\hline Trekkers starting from $2800 \mathrm{~m}^{b}$ & 228 & $86.7 \%$ & 55 & $84.6 \%$ & 83 & $85.6 \%$ & 90 & $89.1 \%$ & .65 \\
\hline Nights to ascend to baseline & 3.4 & \pm .9 & 3.3 & \pm .87 & 3.3 & \pm 1.0 & 3.7 & \pm .9 & .01 \\
\hline Enrolled at $4358 \mathrm{~m}^{c}$ & 151 & $58.5 \%$ & 30 & $46.9 \%$ & 52 & $55.3 \%$ & 69 & $69.0 \%$ & .01 \\
\hline History of altitude illness & 7 & $2.6 \%$ & 3 & $4.6 \%$ & 2 & $2.1 \%$ & 2 & $1.9 \%$ & .55 \\
\hline Positive LLQ score $^{d}$ & 21 & $7.0 \%$ & 7 & $9.2 \%$ & 9 & $8.1 \%$ & 5 & $4.5 \%$ & .40 \\
\hline Enrollment $\mathrm{SaO}_{2}$ & 86.0 & \pm 4.1 & 86.1 & \pm 4.8 & 86.4 & \pm 3.7 & 85.5 & \pm 3.9 & .25 \\
\hline Lost-to-follow up & 78 & $22.7 \%$ & 24 & $27.0 \%$ & 28 & $22.4 \%$ & 26 & $2.2 \%$ & .52 \\
\hline
\end{tabular}

Values are number and percent or mean $\pm \mathrm{SD}$.

${ }^{a}$ Endpoint cohort consists of participants enrolled at baseline and followed up at endpoint $(\mathrm{n}=265)$.

${ }^{b}$ The Lukla airport is at $\sim 2800 \mathrm{~m}$, and trekkers starting from Jiri $(2000 \mathrm{~m})$ pass through Lukla.

${ }^{c}$ Enrollment occurred in the villages of Dingboche $(4358 \mathrm{~m})$ and Pheriche $(4280 \mathrm{~m})$.

${ }^{d}$ Participants were allowed to have 1 mild (LLQ $=1$ ) non-headache symptom on the LLQ to prevent self-selection bias.

medications) revealed several effects not observed in the intent-to-treat evaluation (see Table 3). HAH severity measured by LLQ as moderate or severe had a more frequent incidence in the placebo group as compared to either treatment (acetazolamide $\mathrm{n}=3$ [3.8\%], ibuprofen $\mathrm{n}=4[4.7 \%]$, placebo $\mathrm{n}=7[13.5 \%] ; P=.03$ ). This finding correlated well on the VAS for acetazolamide, while ibuprofen notably was similar to placebo (acetazolamide $2.5 \mathrm{~cm}$, ibuprofen $6.0 \mathrm{~cm}$, placebo $5.9 \mathrm{~cm} ; P=.04$ ). Upon further analysis of VAS headache scores, 3 of the ibuprofen participants recorded outlier VAS scores that were much higher than any values in the acetazolamide group. In small sample sizes outlier scores may bias the results, caution in drawing conclusions based on VAS scores with wide discrepancy in individual values seems prudent.

Multivariate logistic regression analysis was utilized to assess the association of treatment and probability of HAH or AMS incidence as defined by LLQ score. After adjusting for potential baseline confounders (mode of arrival, time spent on ascent to baseline, baseline village altitude, compliance status, age, and sex), no significant difference was found between the treatments for probability of HAH or AMS. However, treatment overall was estimated to reduce the likelihood of development of HAH and AMS compared to placebo (HAH $P=.01$, AMS $P=.02$ ), and results did not vary when excluding noncompliant individuals rather than adjusting for compliance status (data not shown).

\section{Discussion}

This study represents the largest interventional clinical trial for $\mathrm{HAH}$ prevention and establishes 2 important findings. First, acetazolamide and ibuprofen were both shown to be effective in preventing incidence and severity of HAH. Second, ibuprofen performed as well as acetazolamide in the prevention of AMS.

These study data have been externally corroborated in two aspects. First, the protective effect of ibuprofen in development of HAH was found to be roughly similar to the effect of the NSAID aspirin observed in prior smaller studies. ${ }^{15-17}$ Interestingly, acetazolamide was found to be as effective in preventing a pain syndrome (HAH) as the nonsteroidal analgesic ibuprofen. This is not to suggest that acetazolamide is an analgesic; rather it suggests the likelihood that acetazolamide is effectively mitigating the pathophysiologic processes leading to headache pain at altitude.

The secondary finding that ibuprofen performed as well as acetazolamide in the prevention of AMS is novel and will require confirmation. Indirect support of ibuprofen for AMS prophylaxis is implied from multiple studies with either dexamethasone or NSAIDs showing a protective effect against AMS, suggesting that inhibition of the inflammatory cascade may play a significant role in both drug effects. ${ }^{9,20,21,30,31}$

The secondary finding that an approximation of low dose acetazolamide $(255 \mathrm{mg} / \mathrm{d})$ prevents AMS incidence represents the largest trial to date corroborating prior studies. $^{2,3,32}$ This low dose of acetazolamide was found to exhibit a robust and predictable clinical effect.

\section{Theory and Mechanism}

Headache pain in normoxic conditions is thought to originate from activation of the trigeminovascular sys- 


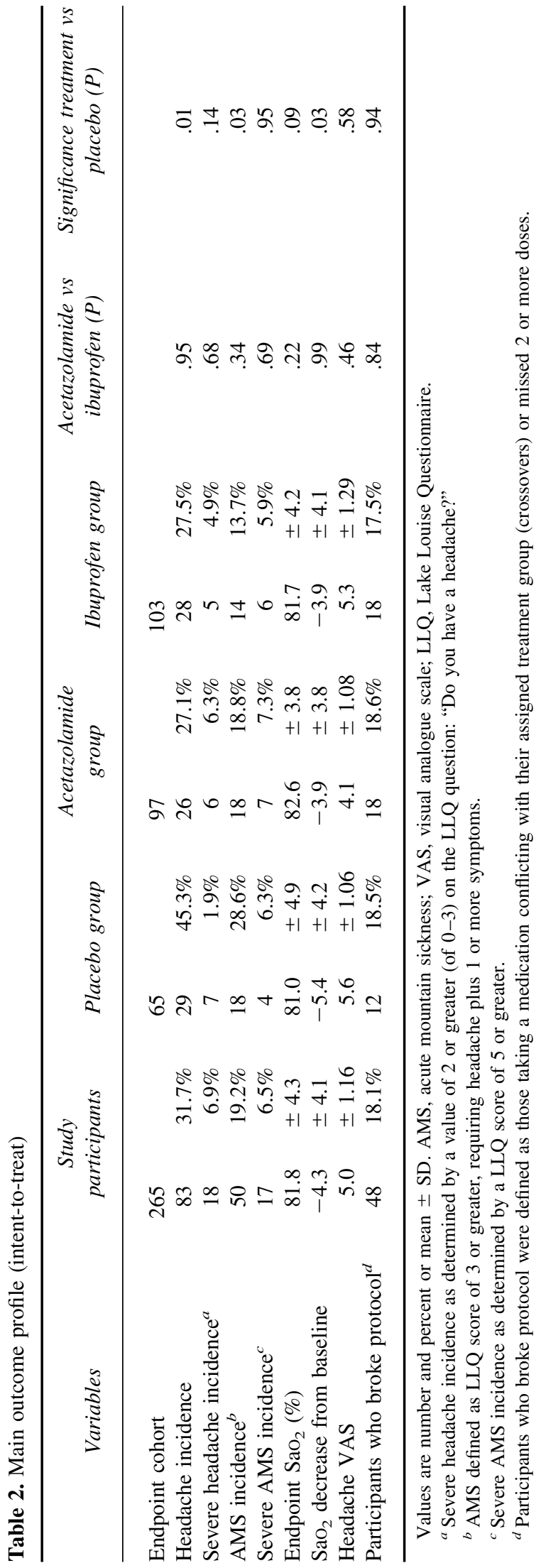

tem by mechanical and/or chemical inflammatory mediators; similar theorized mechanisms of HAH pathophysiology are applied to the action of agents presently studied. ${ }^{11-13}$ An oxygen-starved brain at altitude will swell with blood and create traction on the meninges, potentially irritating mechanoreceptors therein. ${ }^{13}$ Cerebral blood flow has been shown to increase by $26 \%$ upon acute exposure to high altitude, and an increase in cerebral blood volume can be inferred. ${ }^{33}$ This increase in cerebral blood flow is also likely to be associated with distended cerebrovasculature, as observed in transcranial Doppler studies at altitude. ${ }^{34}$

Hypobaric hypoxemia is associated with production of inflammatory mediators known to sensitize meningovascular nociceptors and/or mediate vasodilation. ${ }^{11,12,35-37}$ The inflammatory mediator's role has also been implied by multiple studies using steroids and/or NSAIDs to prevent and treat headaches in the context of AMS and/or high altitude cerebral edema. ${ }^{9,20,21,30,31,35,36}$ However, current evidence suggesting a role for inflammation in the pathophysiology for cerebral forms of acute altitude illness is scant.

Postulated HAH pathophysiology intersects with proposed mechanism of action for both of the study drugs. The basic mechanisms for acetazolamide have already been discussed above. Chemoprophylaxis with the NSAID ibuprofen may be theorized to address HAH pathophysiology by blunting the inflammatory cascade. The implication is that prevention of arachidonic acid metabolism, nociceptor irritation, and vascular endothelial breakdown suggest reasonable mechanisms whereby pain perception and increased cerebral blood flow and edema (leading to downstream events) may be attenuated. ${ }^{11,12,30,38}$

Caution is warranted in interpretation of ibuprofen's efficacy against HAH and AMS. Mechanisms have been discussed that tentatively suggest a means whereby ibuprofen may antagonize the pathophysiology of HAH and AMS. Alternatively, ibuprofen as a simple analgesic may transiently mask painful symptoms of altitude sickness without truly preventing illness. While one quantitative measure in this trial (oxygen saturation) may suggest true protection, this question awaits further careful research of physiologic parameters.

\section{Limitations of the Study}

There are several limitations to application of these data in clinical practice. First, participants had already been exposed to significant altitudes for several days prior to baseline enrollment (4280 or $4358 \mathrm{~m}$ ), a comparatively low-risk setting that could introduce selection bias and limit comparison with other studies. ${ }^{32}$ This study in- 


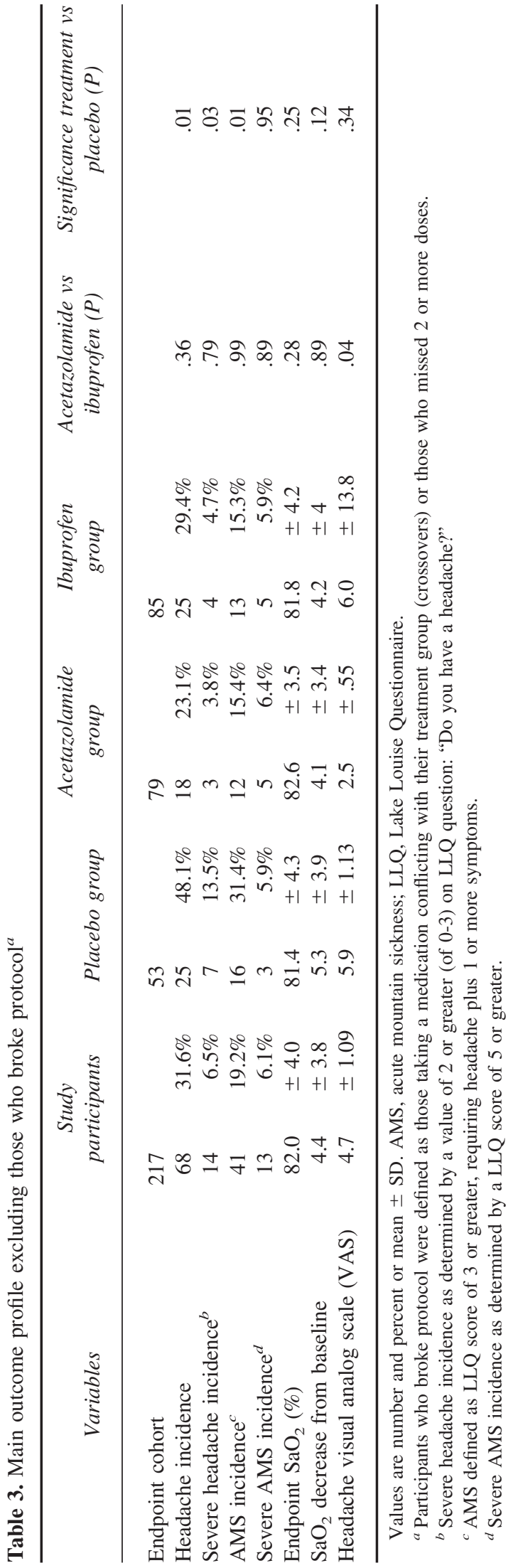

volved a diverse population in typical trekking conditions; these results cannot necessarily be applied to other high altitude trekking environments where ascent rate, demographics, and final elevation may differ. Second, just over a fifth of enrollees were lost to follow-up. Participants in all groups were equally likely to drop out of the study, and some attrition is expected (and similar to prior studies) given the realities of conducting a clinical trial in a wilderness setting. While an intent-to-treat analysis was intended to minimize this bias, it seems plausible that the overall incidence of HAH may be underestimated by these study results. Third, increased sample sizes would permit more power to identify small differences between the treatments, should they exist. This study employed an adequate sample size to detect with $95 \%$ certainty differences between treatments as small as $12 \%$ based on our reasonable prestudy assumptions.

\section{Conclusions}

In summary, these findings are in agreement with prior studies and support the clinical effectiveness of both ibuprofen and acetazolamide in the prevention of HAH. Either medication can be recommended for prophylaxis at altitude, with the inference that acetazolamide may prevent more severe headaches.

The clinical efficacy of low-dose acetazolamide $(\sim 250$ $\mathrm{mg} / \mathrm{d}$ ) in the prevention of AMS has been reconfirmed. Ibuprofen was also found to be well tolerated and effective against symptoms of AMS. This is an interesting finding that implies the possibility of a new prophylactic agent against AMS. Ibuprofen is a commonly used and welltolerated NSAID that could conceivably be used as an alternative to acetazolamide in those susceptible to HAH/AMS and who struggle with a sulfonamide allergy or acetazolamide side effects. However, at present, the widespread use of ibuprofen for HAH and AMS prophylaxis is only cautiously recommended until further research is conducted.

\section{Competing Financial Interests}

No authors received funding or reimbursement for this study from commercial interests.

\section{Council}

This study was conducted under the auspices of the Himalayan Rescue Association and received ethical approval from the Stanford University School of Medicine IRB and the Nepal Health Research Council. 


\section{Acknowledgments}

The authors thank Deurali-Janta Pharmaceuticals of Kathmandu, Nepal and Hari Bhakta Sharma for randomization of the drugs and packaging; Dr Derek and Lydia Lipman for their generous financial support; Vanessa Wilshaw and Mark Thompson for their translations of study materials into Spanish and German, respectively; and the trekkers for their participation.

\section{References}

1. Honigman B, Theis M, Koziol-McLain J, et al. Acute mountain sickness in a general tourist population at moderate altitudes. Ann Intern Med. 1993;118:587-592.

2. Basnyat B, Gertsch JH, Holck PS, et al. Acetazolamide $125 \mathrm{mg}$ BD is not significantly different from $375 \mathrm{mg}$ BD in the prevention of acute mountain sickness: the prophylactic acetazolamide dosage comparison for efficacy (PACE) trial. High Alt Med Biol. 2006;7:17-27.

3. Basnyat B, Gertsch JH, Johnson EW, Castro-Marin F, Inoue Y, Yeh C. Efficacy of low-dose acetazolamide (125 $\mathrm{mg}$ BID) for the prophylaxis of acute mountain sickness. High Alt Med Biol. 2003;4:45-52.

4. Gertsch JH, Basnyat B, Johnson EW, Onopa J, Holck PS, PHAIT. Randomised, double blind, placebo controlled comparison of ginkgo biloba and acetazolamide for prevention of acute mountain sickness among Himalayan trekkers: the prevention of high altitude illness trial (PHAIT). Br Med J. 2004;328:797-799.

5. Silber E, Sonnenberg P, Collier DJ, Pollard AJ, Murdoch DR, Goadsby PJ. Clinical features of headache at altitude: a prospective study. Neurology. 2003;60:1167-1171.

6. Headache Classification Committee of the International Headache Society. The International Classification of Headache Disorders, $2^{\text {nd }}$ Edition. Cephalalgia. 2004;24: 107-108.

7. Serrano-Duenas M. High altitude headache: a prospective study of its clinical characteristics. Cephalalgia. 2005;25: 1110-1116.

8. Hackett PH, Roach RC. High altitude illness. N Engl J Med. 2001;345:107-114.

9. Hackett PH, Roach RC. High altitude cerebral edema. High Alt Med Biol. 2004;5:136-146.

10. Broome JR, Stoneham MD, Beeley JM, Milledge JS, Hughs AS. High altitude headache: treatment with ibuprofen. Aviat Space Environ Med. 1994;65:19-20.

11. Strassman AM, Leavy D. Response properties of dural nociceptors in relation to headache. J Neurophysiol. 2006; 95:1298-1306.

12. Strassman AM, Raymond SA, Burstein R. Sensitization of meningeal sensory neurons and the origin of headaches. Nature. 1996;384:560-564.

13. Sanchez Del Rio M, Moskowitz M. High altitude headache: lessons from headaches at sea level. Hypoxia: into the next millenium. Adv Exp Med Biol. 1999; 474:145-153.
14. Hersh EV, Moore PA, Ross GL. Over the counter analgesics and antipyretics: a critical asessment. Clin Ther. 2000; 22:500-524.

15. Burtscher M, Likar R, Nachbauer W, Philadelphy M, Puhringer R, Lammle T. Effects of aspirin during exercise on the incidence of high altitude headache: a randomized, double-blind, placebo-controlled trial. Headache. 2001;41: 542-545.

16. Burtscher M, Philadelphy M, Likar R. Aspirin versus diamox plus aspirin for headache prevention during physical activity at high altitude. Adv Exp Med Biol. 1999;474: 370-371.

17. Burtscher M, Likar R, Nachbauer W, Philadelphy M. Aspirin for prophylaxis against headache at high altitudes: randomised, double blind, placebo controlled trial. $\mathrm{Br}$ Med J. 1998;316:1057-1058.

18. Meehan RT, Cymerman A, Rock P, et al. Effect of naproxen on acute mountain sickness and vascular responses to hypoxia. Am J Med Sci. 1986;292:15-20.

19. Kayser B, Hulsebosch R, Bosch F. Low-dose acetylsalicylic acid analog and acetazolamide for prevention of acute mountain sickness. High Alt Med Biol. 2008;9:15-23.

20. Albin K, Kanaan N, Goodblatt M, Powell F. Surveying physiological factors associated with acute mountain sickness on White Mountain Peak. Abstract \#126. Presented at the 16th International Hypoxia Symposium; Lake Louise, Alberta, Canada; March 10-15, 2009.

21. Dumont L, Mardirosoff C, Tramer MR. Efficacy and harm of pharmacological prevention of acute mountain sickness: quantitative systematic review. Br Med J. 2000;321:267-272.

22. Carrion E, Hertzog JH, Medlock MD, Hauser GJ, Dalton HJ. Use of acetazolamide to decrease cerebrospinal fluid production in chronically ventilated patients with ventriculopleural shunts. Arch Dis Child. 2001;84:68-71.

23. Leaf DE, Goldfarb DS. Mechanisms of action of acetazolamide in the prophylaxis and treatment of acute mountain sickness. J Appl Physiol. 2007;102:1313-1322.

24. World Medical Association. Declaration of Helsinki: ethical principles for medical research involving human subjects. http://www.wma.net/e/policy/b3.htm. Accessed August 21, 2005.

25. Roach RC, Bartsch P, Hackett PH. The Lake Louise Acute Mountain Sickness Scoring System. In: Sutton JR, Houston CS, Coates G, eds. Hypoxia and Molecular Medicine: Proceedings of the 8th International Hypoxia Symposium. Burlington, VT: Queen City Printers; 1993:272-274.

26. Bartsch P, Muller A, Hofstettler D, Maggiorini M, Vock P, Oelz O. AMS and HAPE scoring in the Alps. In: Sutton JR, Houston CS, Coates G, eds. Hypoxia and Molecular Medicine: Proceedings of the 8th International Hypoxia Symposium. Burlington, VT: Queen City Printers; 1993;265-271.

27. Todd KH, Funk JP. The minimum clinically important difference in physician-assigned visual analog pain scores. Acad Emerg Med. 1996;3:142-146.

28. Gallagher EJ, Liebman M, Bijur PE. Prospective validation of clinically important changes in pain severity measured 
on a visual analog scale. Ann Emerg Med. 2001; 38:633-638.

29. R Development Core Team. R: A language and environment for statistical computing. R Foundation for Statistical Computing, Vienna, Austria. ISBN 3-900051-07-0; 2007. http://www.R-project.org. Accessed May 8, 2007.

30. Levy D, Zhang X, Jakubowski M, Burstein R. Sensitization of meningeal nociceptors: inhibition by naproxen. Eur J Neurosci. 2008;27:917-922.

31. Montgomery AB, Luce JM, Michael P, Mills J. Effects of dexamethasone on the incidence of acute mountain sickness at two intermediate altitudes. JAMA. 1989;261:734-736.

32. van Patot MC, Leadbetter G 3rd, Keyes LE, Maakestad KM, Olson S, Hackett PH. Prophylactic low-dose acetazolamide reduces the incidence and severity of acute mountain sickness. High Alt Med Biol. 2008;9:289-293.

33. Severinghaus JW, Chiodi H, Eger EI, Brandstater B, Hornbein TF. Cerebral blood flow in man at high altitude: Role of cerebrospinal fluid $\mathrm{pH}$ in normalization of flow in chronic hypocapnia. Circ Res. 1966;19:274-282.

34. Ter Minassian A, Beydon L, Ursino M, Gardette B, Gortan C, Richalet JP. Doppler study of middle cerebral artery blood flow velocity and cerebral autoregulation during a simulated ascent of Mount Everest. Wilderness Environ Med. 2000;12:175-183.

35. Hartmann G, Tschöp M, Fischer R, et al. High altitude increases circulating interleukin-6, interleukin-1 receptor antagonist, and C-reactive protein. Cytokine. 2000;12: 246-252.

36. Maggiorini M, Streit M, Siebenmann C, et al. Dexamethasone decreases systemic inflammatory and stress response and favors vasodilation in high altitude pulmonary edema susceptible at $4559 \mathrm{~m}$. Abstract presented at the $16^{\text {th }}$ International Hypoxia Symposium; Chateau Lake Louise, Alberta, Canada; March 10-15, 2009.

37. Richalet JP, Hornych A, Rathat C, Aumont J, Larmignat P, Rémy P. Plasma prostaglandins, leukotrienes, and thromboxane in acute high altitude hypoxia. Respir Physiol. 1991;85:205-215.

38. Buzzi MG, Sakas DE, Moskowitz MA. Indomethacin and acetylsalicylic acid block neurogenic plasma protein extravasation in rat dura mater. Eur J Pharm. 1989;165: 251-258. 